DOI: $10.17957 / \mathrm{IJAB} / 15.1766$

http://www.fspublishers.org

\title{
Antifungal Activity of Root Extracts from Baccharis salicina on Germination of Uredospores of Hemileia vastatrix
}

\author{
Anayancy Lam-Gutiérrez ${ }^{1,4}$, Robert Winkler², Eduardo Raymundo Garrido-Ramírez ${ }^{3}$, Reiner Rincón-Rosales ${ }^{1}$, \\ Federico Antonio Gutiérrez-Miceli ${ }^{1}$, Betsy Anaid Peña-Ocaña ${ }^{1}$, Jorge Martín Guzmán-Albores ${ }^{1}$ and Víctor Manuel \\ Ruíz-Valdiviezo ${ }^{1^{*}}$ \\ ${ }^{1}$ Tecnológico Nacional de Mexico/IT de Tuxtla Gutiérrez, Carretera Panamericana km 1080, C.P. 29050, Tuxtla Gutiérrez, \\ Chiapas, México \\ ${ }^{2}$ Centro de Investigación y Estudios Avanzados del Instituto Politécnico Nacional, Km. 9.6 Libramiento Norte Carretera \\ Irapuato-León 36824 Irapuato Gto. México \\ ${ }^{3}$ INIFAP, Campo Experimental Centro de Chiapas, Carretera Ocozocoautla-Cintalapa km 3, Ocozocoautla, Chiapas, México \\ C.P. 29140, Chiapas, Mexico \\ ${ }^{4}$ Tecnológico Nacional de Mexico/IT Superior de Cintalapa, Carretera Panamericana km 995, C.P. 30400, Cintalapa, \\ Chiapas, México
}

*For correspondence: bioqvic@hotmail.com

Received 04 November 2020; Accepted 25 January 2021; Published 16 April 2021

\begin{abstract}
Hemileia vastatrix is a fungus associated with coffee leaf rust, the most destructive disease of Coffea arabica. The objective of this work was to evaluate the antifungal activity of alcoholic extracts from roots of Baccharis salicina and to determine the metabolites present in these fractions. Antifungal activity was evaluated under in vitro conditions by monitoring the germination ability of $H$. vastatrix, the coffee leaf rust pathogen. In order to determine the presence of metabolites, chemical characterization of fractions obtained from methanolic root extracts was performed with help of an untargeted metabolomic approach and by using high-resolution mass spectrometry (MS) and MS ${ }^{2}$ based on directinjection electrospray mass spectrometry (DIESI-MS) and hierarchical cluster analysis (HCA). Germination percentage was evaluated by leaves fixation technique. The MEBs significantly decreased the percentage of germination of $H$. vastatrix to levels below $5 \%$ as the dose increased. The multivariable analysis confirmed that the distribution of three fractions of methanolic extracts belonged to polyketides, organoheterocyclic compounds, fatty acyls, prenol-lipids, organo-oxygen and farnesene classes. This report comprises the first study of the metabolomic profile and biological activity study of roots from B. salicina against coffee leaf rust pathogen $H$. vastatrix. (C) 2021 Friends Science Publishers
\end{abstract}

Keywords: Antifungal activity; MS-MS analysis; Hemileia vastatrix; Multivariate analysis; Baccharis salicina

\section{Introduction}

Asteraceae is regarded as the largest family of widely distributed flowering plants in the world, being absent only in Antarctica. This family contains mostly herbaceous plants and small shrubs and rarely trees, with an estimated 23,600 species belonging to 1600 genera with 12 subfamilies and 28 tribes (Ramos et al. 2016). The Astereae tribe includes the subtribe Baccharidinae Less., which is exclusively on the American continent and includes the genera Archibaccharis and Baccharis. Baccharis plants are usually studied due to their biological activities such as antibacterial, antifungal, antiprotozoal, antiviral, antioxidant, antidiabetic, immunomodulatory, antimutagenic and chemopreventive properties (Abad and Bermejo 2007).
In Southern México, Baccharis salicina, is known as chilcanka' or chilca in the Tzotzil language. In this region, it has been used as a traditional medicinal plant. The locals use infusions from these species' leaves to treat gynecological and digestive disorders (Abad and Bermejo 2007; Ramos et al. 2016). Methanolic extracts of its leaves contain antifungal properties against phytopathogens, but few reports regarding the chemical composition of the plant are available. It has been reported that methanolic extracts of its leaves contain antifungal properties against phytopathogens. In this regard, significant effects on the growth of mycotoxigenic fungi have been found (Tequida-Meneses et al. 2002; RosasBurgos et al. 2011). Likewise, the methanolic extract from roots has reportedly inhibited sporulation and mycelial growth of Aspergillus ochraceus and Fusarium moniliforme.

\footnotetext{
To cite this paper: Lam-Gutiérrez A, R Winkler, ER Garrido-Ramírez, R Rincón-Rosales, FA Gutiérrez-Miceli, BA Peña-Ocaña, JM Guzmán-Albores, VM Ruíz-
} Valdiviezo (2021). Antifungal activity of root extracts from Baccharis salicina on germination of uredospores of Hemileia vastatrix. Intl J Agric Biol 25:1075-1084 
This was reported for the first time in a chemical composition study by GC-MS (Lam-Gutiérrez et al. 2019). Thus, due to the importance of this genus, phytochemical studies and the search for new active molecules of more Baccharis species offer promising outcomes.

Coffee leaf rust, the most destructive disease in the cultivation of coffee worldwide, is caused by the fungus $H$. vastatrix, mainly affecting $C$. arabica, which represents an estimated $70 \%$ of the production worldwide (Talinhas et al. 2016). $H$. vastatrix is an obligate biotrophic fungus, penetrating and sporulating through stomata (López-Bravo et al. 2012). The need to have less toxic and more environment friendly fungicides is a challenge when it comes to searching novel antifungal agents (Naqvi et al. 2019; Javaid et al. 2020). Nowadays, the finding relies mainly on ethnobotanical information and exploration of new bioactive compounds obtained from native plants (Yoon et al. 2013; Akhtar et al. 2020), which are increasingly being studied in a holistic way. Thus, omics approaches are being used to provide qualitative and quantitative descriptions. Metabolomics is a wellestablished research field that is developing rapidly for the study of metabolites, relying on fast and broad screening (Cox et al. 2014) and supported by modern instruments. These instruments include mass spectrometry (MS), which has allowed the detection of many chemical compounds with high sensitivity and precision, thanks to direct-injection electrospray mass spectrometry (DIESI-MS). This technology has proven to be an important tool in the high throughput of non-targeted metabolite fingerprint (GarcíaFlores et al. 2015). Therefore, the objective of this study was to evaluate the inhibitory effect of $B$. salicina root extracts on the germination of coffee rust (H. vastatrix) uredospores and to identify the composition of the most abundant metabolites in MEBs through use of DIESI-MS and $\mathrm{MS}^{2}$.

\section{Materials and Methods}

\section{Plant material}

Plants were collected in Chiapas, México, in San Cristóbal de las Casas (coordinates 16 $6^{\circ} 5^{\prime} \mathrm{N}$ and $92^{\circ} 38^{\prime} \mathrm{O}$ and 2255 $\mathrm{m}$ altitude), where Mexican pine-oak forest vegetation and a temperate climate predominates. The $B$. salicina was authenticated and registered under number 49985 at the herbarium of Dr. Faustino Miranda Botanical Garden, in Chiapas, Mexico.

\section{Methanolic extracts of roots from $B$. salicina (MEBs)}

The roots of $B$. salicina were dried at room temperature, ground to a fine powder, and sifted using a $2 \mathrm{~mm}$ mesh sieve. Subsequently, $300 \mathrm{~g}$ of pulverized roots were macerated in $3 \mathrm{~L}$ of methanol, as reported by Lam-Gutiérrez et al. (2019).

\section{Fraction preparation of MEBs}

As an analysis approach, the MEBs were partitioned into three fractions. MEBs were suspended in hexane and the soluble organic layer was separated and concentrated until drying under vacuum conditions at $55^{\circ} \mathrm{C}$ (hexane fraction). The insoluble residue was suspended in ethyl acetate, after which the soluble organic layer was removed and treated under vacuum conditions in order to concentrate the ethyl acetate fraction at $55^{\circ} \mathrm{C}$. Finally, the last insoluble residue was the methanolic fraction. Phytochemical composition of hexane, ethyl acetate and methanol fractions were analyzed by spectrometer mass.

\section{Mass spectrometry (MS) and tandem mass spectrometry $\left(\mathbf{M S}^{2}\right)$}

Screening tests of spectrum mass analysis were carried out using a mass analyzer quadrupole Micromass ZQ 2000 Waters $\circledR$. For all measurements, ionization by electrospray (ESI) was used, both in positive and negative modes. The voltages for the ionization source were established at $3 \mathrm{KV}$ for the capillary, $60 \mathrm{~V}$ for cone and $3 \mathrm{~V}$ at the extractor. The $\mathrm{RF}$ lens (orientation range-finding) was used at $0.5 \mathrm{~V}$. Temperatures were set to $80^{\circ} \mathrm{C}$ and $150^{\circ} \mathrm{C}$ for the source and the desolvation, respectively. The used gas was nitrogen with a flow of $250 \mathrm{~L} / \mathrm{h}$ for desolvation and $50 \mathrm{~L} / \mathrm{h}$ for cone. Multiplier was set to a value of $650 \mathrm{~V}$. The spectra were obtained continuously in a range of 50-2000 m/z. Each run had duration of $1 \mathrm{~min}$, with a scan time of $10 \mathrm{~s}$ and an interscan time of $0.1 \mathrm{~s}$. The samples were injected at a rate of 10 $\mu \mathrm{L} / \mathrm{min}$. The fragmentation of the molecules $\left(\mathrm{MS}^{2}\right)$ with the highest concentration in each fraction obtained was performed with the LCQ FleetTM Ion Trap Thermo Scientific ion trap mass spectrometer, with 6 microscans, at $300^{\circ} \mathrm{C}$ capillary temperature, $35 \mathrm{~V}$ capillary voltage, 4.42 $\mathrm{kV}$ spray voltage, $45 \mathrm{~V}$ tube lens voltage and 30 units of nitrogen gas (AU) with ionization energies from 20 to 40 $\mathrm{eV}$, in positive and negative mode.

\section{Raw data processing}

A revision of the mass spectra was obtained via a bioinformatic analysis. Alignment and correction of the baseline was done by extracting data relating $\mathrm{m} / \mathrm{z}$ and relative intensity by use of $\mathrm{M}$ mass V. 5.5.0 software to generate the study matrix. Subsequently, the Metaboanalyst platform version 4.0 was used, with functions written in $\mathrm{R}$ software version 3.4 .3 for multivariate statistical analysis (Xia and Wishart 2016).

The putative identification of metabolites was crossreferenced against information related to monoisotopic mass, mass spectra, most probable molecular mass and a mechanism for breaking-fragmentation patterns in online libraries (ChemSpider, HMDB, LIPID MAPS, MassBank, MetFrag, METLIN, PubMed, SpiderMass). 


\section{Fungi}

Samples of rust (H. vastatrix) were isolated from the $C$. arabica var. Bourbon located in Ocuilapa, Municipality of Ocozocoutla, Chiapas, Mexico, with the coordinates $16^{\circ} 52^{\prime} 22.46^{\prime} \mathrm{N}$ and $93^{\circ} 24^{\prime} 39.39^{\prime} \mathrm{W}$. Previously, the presence of the fungus was qualitatively identified on infected leaves for a characteristic yellow or orange powder, reniform, smooth on the inside and rough on the outside (Fig. 1; McCain and Henen 1984). The uredospores were scraped from the surface of the collected leaves and stored at $4^{\circ} \mathrm{C}$ until ready to be used.

\section{Separate leaf test}

A suspension of $1 \times 10^{5}$ uredospores of $H$. vastatrix in $50 \mathrm{~mL}$ of distilled water was prepared previously, which also included a drop of Tween 20. Afterwards, healthy and young leaves from $C$. arabica plants were cut and immersed for $3 \mathrm{sec}$ in MEBs at 100,270 and $750 \mathrm{mg} \mathrm{mL}^{-1}$, respectively. They were then placed in Petri dishes with a base of wet filter paper, and the undersides of the exposed leaves were inoculated by dripping $1 \mathrm{~mL}$ of the previously obtained spore suspension. Petri dishes were kept in darkness for $24 \mathrm{~h}$. Subsequently, they underwent a 12/12 photoperiod at $22^{\circ} \mathrm{C}$ and $65 \%$ relative humidity. Monitoring of the Petri dishes occurred at 4, 8, 14, 20.5, 32.5, 45.5, 69.5 and $165.5 \mathrm{~h}$ after inoculation, which consisted on counting the germinated spores fixated on the leaves. Each coffee leaf was cut from the central part into pieces of approximately 1 $\mathrm{cm}^{2}$. The pieces were then collected in a beaker and added 5 $\mathrm{mL}$ of Carnoy solution, and let to incubate for $4 \mathrm{~h}$ at $60^{\circ} \mathrm{C}$.

Subsequently, the Carnoy solution was drained, $5 \mathrm{~mL}$ of lactophenol solution were added to the fragment, and let for a $5 \mathrm{~h}$ incubation at $60^{\circ} \mathrm{C}$. After this time, the lactophenol was strained off of the leaf fragments; the fragments were stained with $0.2 \%$ trypan blue and left at room temperature for $1.5 \mathrm{~h}$, then washed with sterile distilled water until the dye was completely washed off. Smears of the washed fragments were placed on each slide with two drops of polyvinyl alcohol, then covered with slips, and let to dry at room temperature.

The same procedure was carried out for each sample. The number of germinated spores was counted at each monitoring time with help of a Primo StarZeiss microscope and a Canon G10 digital camera.

\section{Statistical analysis}

The experiments were performed by following a completely randomized design with 3 treatments and three replicates. In addition, two controls were including, one without application of fungicide (negative control) and another with the application of the commercial fungicide used by the producers (positive control). Statistical analyses consisted of an analysis of variance (ANOVA) with $\alpha=0.05$ and Tukey test was used to determine the statistically different treatments, with a total of 144 experimental units. This was conducted using the Statgraphic Centurion version XV software.

\section{Results}

The experimental tests in vitro for antifungal activity allowed the observation of the infective process of the uredospore from $H$. vastatrix on leaves of $C$. arabica var. Bourbon. According to Table 1 , in the positive control treatment B (application of inoculum only on leaves) a 15\% of spore germination demonstrated after $32.5 \mathrm{~h}$.

MEBs proved to have an inhibitory effect on the germination percentages of uredospores (Table 1). It was found that, regardless of the sampling time, as that of $32.5 \mathrm{~h}$, leaves treated with MEBs significantly decreased the percentage of germination by $<5 \%$ as the dose increased $(P$ $<0.05)$.

\section{Antifungal activity: germination percentage}

The application of MEBs at concentrations of 250 and 750 $\mathrm{mg} \mathrm{mL}^{-1}$ allowed to obtain the highest inhibition percentage of spore germination compared with copper oxychloride used as negative control (Table 1). The latter is actually the most common chemical treatment to prevent leaf rust disease spreading on Coffee arabica crops throughout the American continent. In the compatible interactions between plant and uredospores, a penetration hypha differentiates from an appressorium and develops into the substomatal chamber. At the growing tip of the penetration hypha, two thick lateral branches are formed (anchor), and differentiate into a haustorial mother cell (HMC), which gives rise to haustorium in the stomata subsidiary cells prior to the formation of haustoria in mesophyll cells. This is a unique feature of $H$. vastatrix.

\section{Mass spectrum}

In order to know the main chemical components in MEBs with likely antifungal potential, an analytical strategy was proposed for improving their study. The strategy consisted of partition in the chemical components into three fractions, according to their polarity. Fractioning was achieved with use of the following solvents: ethyl acetate, hexane and methanol. In the pre-analytical sample preparation, before instrumental analysis, an important step was carried out to increase detection of low-abundance from roots plant metabolites. Two of the fractions were dissolved once again in a compatible solvent with the ion trap spectrometer aiming to obtain the highest intensity detection. The hexane fraction was dissolved in $\mathrm{ACN} / \mathrm{MeOH}$ (50:50), the ethyl acetate fraction in $\mathrm{ACN}$, and the methanolic fraction remained suspended in the organic solvent without alterations. Two features viz., 5386 and 1189 in ESI (+) and (-) ionization mode, respectively, were obtained after 
Table 1: In vitro: Effect of MEBg on the germination capacity of uredospores from $H$. vastatrix. Tukey test $(P<0.05)$. The uppercase letters indicate fixed leaves: A) Without inoculum and treatment; B) Positive control (Inoculum); C) Negative control: copper oxychloride; D) $100 \mathrm{mg} \mathrm{mL}^{-1}$; E) $270 \mathrm{mg} \mathrm{mL}^{-1}$; F) $750 \mathrm{mg} \mathrm{mL}^{-1}$

\begin{tabular}{lllllllll}
\hline Treatment & \multicolumn{7}{c}{ Time (h after inoculation) } \\
\cline { 2 - 9 } & 4 & 8 & 14 & 20.5 & 32.5 & 45.5 & 69.5 & 165.5 \\
\hline $\mathrm{A}$ & $0.00^{\mathrm{a}}$ & $0.00^{\mathrm{a}}$ & $0.00^{\mathrm{a}}$ & $0.00^{\mathrm{a}}$ & $0.00^{\mathrm{a}}$ & $0.00^{\mathrm{a}}$ & $0.00^{\mathrm{a}}$ & $0.00^{\mathrm{a}}$ \\
$\mathrm{B}$ & $0.00^{\mathrm{a}}$ & $0.00^{\mathrm{a}}$ & $0.00^{\mathrm{a}}$ & $0.69^{\mathrm{b}}$ & $14.86^{\mathrm{b}}$ & $21.66^{\mathrm{b}}$ & $37.52^{\mathrm{b}}$ & $37.35^{\mathrm{b}}$ \\
$\mathrm{C}$ & $1.33^{\mathrm{b}}$ & $0.00^{\mathrm{a}}$ & $4.44^{\mathrm{b}}$ & $0.90^{\mathrm{b}}$ & $2.75^{\mathrm{c}}$ & $3.83^{\mathrm{c}}$ & $23.21^{\mathrm{c}}$ & $38.54^{\mathrm{b}}$ \\
$\mathrm{D}$ & $1.95^{\mathrm{b}}$ & $1.51^{\mathrm{b}}$ & $1.92^{\mathrm{b}}$ & $2.48^{\mathrm{c}}$ & $4.45^{\mathrm{c}}$ & $3.52^{\mathrm{c}}$ & $16.43^{\mathrm{c}}$ & $7.01^{\mathrm{c}}$ \\
$\mathrm{E}$ & $2.29^{\mathrm{b}}$ & $0.00^{\mathrm{a}}$ & $0.00^{\mathrm{a}}$ & $1.23^{\mathrm{bc}}$ & $0.00^{\mathrm{a}}$ & $1.33^{\mathrm{cd}}$ & $5.03^{\mathrm{d}}$ & $1.67^{\mathrm{d}}$ \\
$\mathrm{F}$ & $2.56^{\mathrm{b}}$ & $0.00^{\mathrm{a}}$ & $0.00^{\mathrm{a}}$ & $8.98^{\mathrm{c}}$ & $3.61^{\mathrm{c}}$ & $0.00^{\mathrm{a}}$ & $4.32^{\mathrm{d}}$ & $2.20^{\mathrm{d}}$ \\
\hline \multicolumn{2}{l}{ Latin letters a, b, c, d (superscript) in the same column indicates significant differences } & &
\end{tabular}

Table 2: Significant compounds detected in roots of Baccharis salicina by DIESI-MS, positive mode

\begin{tabular}{|c|c|c|c|c|c|c|c|c|}
\hline \multicolumn{9}{|c|}{ The most intense compounds in Baccharis glutinosa roots } \\
\hline Fraction & $\begin{array}{l}\mathrm{m} / \mathrm{z}[\mathrm{m}+\mathrm{H}] \\
+(\text { ionization } \\
\text { mode })\end{array}$ & Fragment pattern $[\mathrm{m}+\mathrm{H}]^{+}\left(\mathrm{MS}^{2}\right)$ & $\begin{array}{l}\text { Ionization } \\
\text { energy (V) }\end{array}$ & $\begin{array}{l}\text { Molecular } \\
\text { formula }\end{array}$ & Putative compound & Class & $\begin{array}{l}\text { Data } \\
\text { base }\end{array}$ & ID data base \\
\hline \multirow[t]{5}{*}{ Ethyl acetate } & $284.1249(+)$ & $228.17,157.17,170.08,198$ & 35 & $\mathrm{C}_{17} \mathrm{H}_{32} \mathrm{O}_{3}$ & 5-(1-hydroxytridecyl)oxolan-2-one & Fatty Acyls & Metlin & 93340 \\
\hline & $380.3365(+)$ & $\begin{array}{l}252.25,266.25,238.50,280.25, \\
308.25,197.25\end{array}$ & 35 & $\mathrm{C}_{22} \mathrm{H}_{20} \mathrm{O}_{6}$ & Multijuginol & $\begin{array}{l}\text { Polyketides: } \\
\text { flavonoids }\end{array}$ & Metlin & 48617 \\
\hline & $174.0679(+)$ & $156.17,97.92,115.92,132.08,86$ & 25 & $\mathrm{C}_{9} \mathrm{H}_{18} \mathrm{O}_{3}$ & 3-hydroxy-nonanoic acid & Fatty Acyls & Metlin & 35407 \\
\hline & $156.8757(+)$ & $\begin{array}{l}138.17, \quad 113.83, \quad 74.00, \quad 97.92, \\
55.08,128\end{array}$ & 30 & $\mathrm{C}_{6} \mathrm{H}_{4} \mathrm{O}_{5}$ & 2,5-Furandicarboxylic acid & $\begin{array}{l}\text { Organoheterocycli } \\
\mathrm{c} \text { compounds: } \\
\text { furans }\end{array}$ & MoNA & $\begin{array}{l}\text { Spectrum } \\
\text { HMDB0004812 } \\
\text { ms_ms_2403 }\end{array}$ \\
\hline & $190.9425(+)$ & $174.17,156.00$ & 25 & $\mathrm{C}_{6} \mathrm{H}_{8} \mathrm{O}_{7}$ & $\begin{array}{l}\text { Glucaric acid lactone; (2S)-[(2S,3R,4R)- } \\
\text { 3,4-dihydroxy-5-oxotetrahydrofuran-2- } \\
\text { yl](hydroxy)ethanoic acid }\end{array}$ & conjugate acid & Metlin & 44758 \\
\hline \multirow[t]{3}{*}{ Hexane } & $380.9073(+)$ & $252.17,325.17,366.33,123$ & 30 & $\mathrm{C}_{22} \mathrm{H}_{20} \mathrm{O}_{6}$ & Multijuginol & $\begin{array}{l}\text { Polyketides: } \\
\text { flavonoids }\end{array}$ & Metlin & 48617 \\
\hline & $240.0411(+)$ & $\begin{array}{l}143.08,97.17,222.08,183.08, \\
80.83\end{array}$ & 30 & $\mathrm{C}_{15} \mathrm{H}_{12} \mathrm{O}_{3}$ & $\begin{array}{l}\text { (Z)-3-hydroxy-1-(2-hydroxyphenyl)-3- } \\
\text { phenylprop-2-en-1-one; tambiéns se llama } \\
\text { 2'-beta-Dihydroxychalcone }\end{array}$ & $\begin{array}{l}\text { polyketides: } \\
\text { flavonoids: } \\
\text { chalcones }\end{array}$ & Metlin & 43644 \\
\hline & $333.0255(+)$ & $96.75,309.33 .180 .00$ & - & - & - & - & - & - \\
\hline \multirow[t]{5}{*}{ Methanolic } & $381.0341(+)$ & $252.17,325.17,366.33,123$ & 35 & $\mathrm{C}_{22} \mathrm{H}_{20} \mathrm{O}_{6}$ & Multijuginol & $\begin{array}{l}\text { Polyketides: } \\
\text { flavonoids }\end{array}$ & Metlin & 48617 \\
\hline & $218.8543(+)$ & $\begin{array}{l}\text { 111, 147.17, 159.17, 204.08, } \\
135.08,119\end{array}$ & 30 & $\mathrm{C}_{15} \mathrm{H}_{22} \mathrm{O}$ & $\begin{array}{l}\text { Dendrolasin } \\
\text { nonadienyl)-Furan) }\end{array}$ & Farnesenes & Metlin & 71335 \\
\hline & $365.1163(+)$ & $203.08,184.92$ & 25 & $\mathrm{C}_{14} \mathrm{H}_{17} \mathrm{NO}_{9}$ & $\begin{array}{l}\text { (2S)-7-Hydroxy-3-oxo-3,4-dihydro-2H- } \\
\text { 1,4-benzoxazin-2-yl beta-D- } \\
\text { glucopyranoside }\end{array}$ & $\begin{array}{l}\text { O-glycosyl } \\
\text { compounds }\end{array}$ & MoNA & $\begin{array}{l}\text { Spectrum } \\
\text { CCMSLIB00000 } \\
849622\end{array}$ \\
\hline & $332.8352(+)$ & $240.25,296.42,270.42,254.08$ & 30 & $\mathrm{C}_{19} \mathrm{H}_{24} \mathrm{O}_{5}$ & Gibberellin A51 & $\begin{array}{l}\text { Prenol-lipids: } \\
\text { isoprenoids }\end{array}$ & Metlin & 41234 \\
\hline & $155.8606(+)$ & 111.92 & 30 & - & - & - & - & - \\
\hline
\end{tabular}

alignment and integration correction of the set of samples fingerprint by DIESI-MS. The data matrix was used for statistical evaluation. Representative peaks of samples in positive mode are shown in Fig. 2.

The MEBs were found to have various functional groups of compounds. The most abundant putative secondary metabolites identified (DIESI-MS in positive mode) were polyketides (flavonoids), such as multijuginol with the peak corresponding to $380 \mathrm{~m} / \mathrm{z}$ in ethyl acetate and hexane fractions, 2'-beta-Dihydroxychalcone with peak at $240.041 \mathrm{~m} / \mathrm{z}$ in hexane fraction, these were followed by furans, such as 2,5-Furandicarboxylic acid in ethyl acetate fraction (Fig. 2 and Table 2). On the other hand, the most abundant compounds identified (DIESI-MS in negative mode) were polyketides (flavonoids), such as 3'methoxyquercetinwith the peak corresponding to $316(-) \mathrm{m} / \mathrm{z}$ in ethyl acetate fraction, 12a $\beta$-hydroxydeguelin with its peak at 411(-) $\mathrm{m} / \mathrm{z}$ in hexane fraction and 3,4dihydroxycinnamic acid with the peak at $179(-) \mathrm{m} / \mathrm{zin}$ methanolic fraction (Table 3).

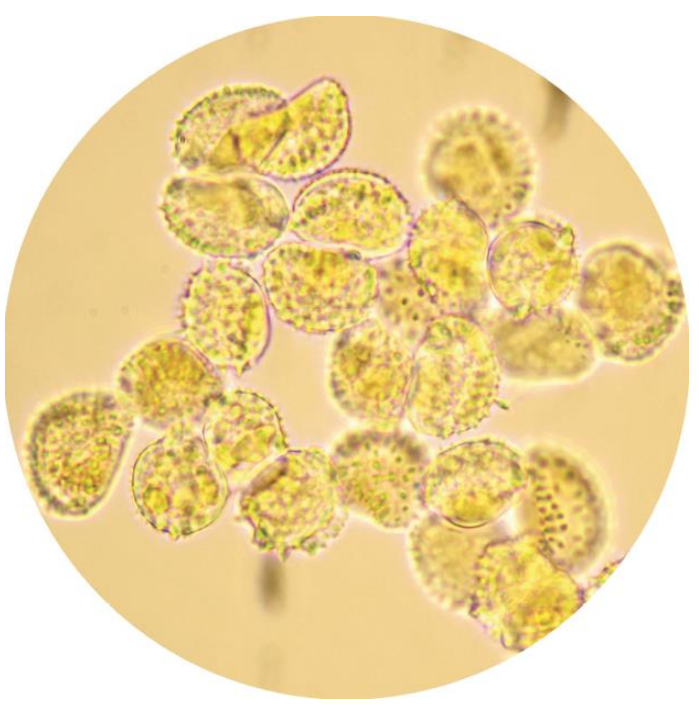

Fig. 1: Uredospores of $H$. vastatrix, contrast microscope: Objective 100X. Canon camera G10 

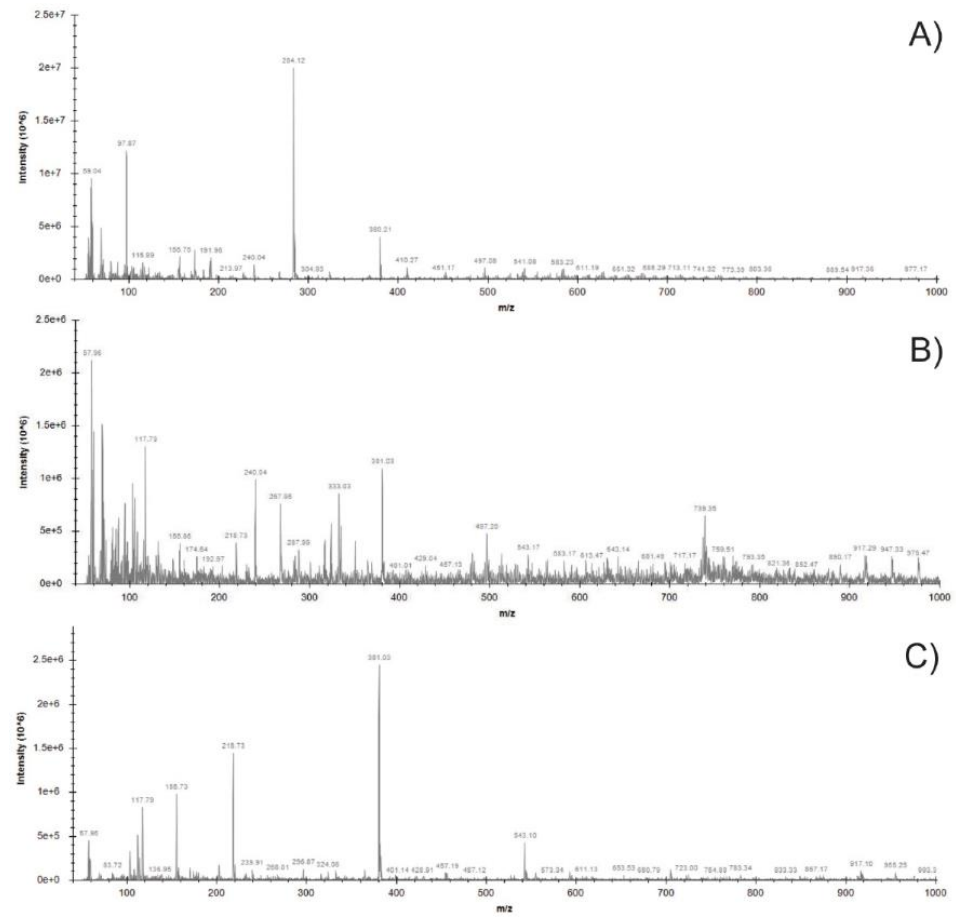

Fig. 2: Mass spectrum of MEBs partitioned: A) Ethyl acetate, B) Hexane and C) Methanolic fractions. Acquired with DIESI-MS in positive mode

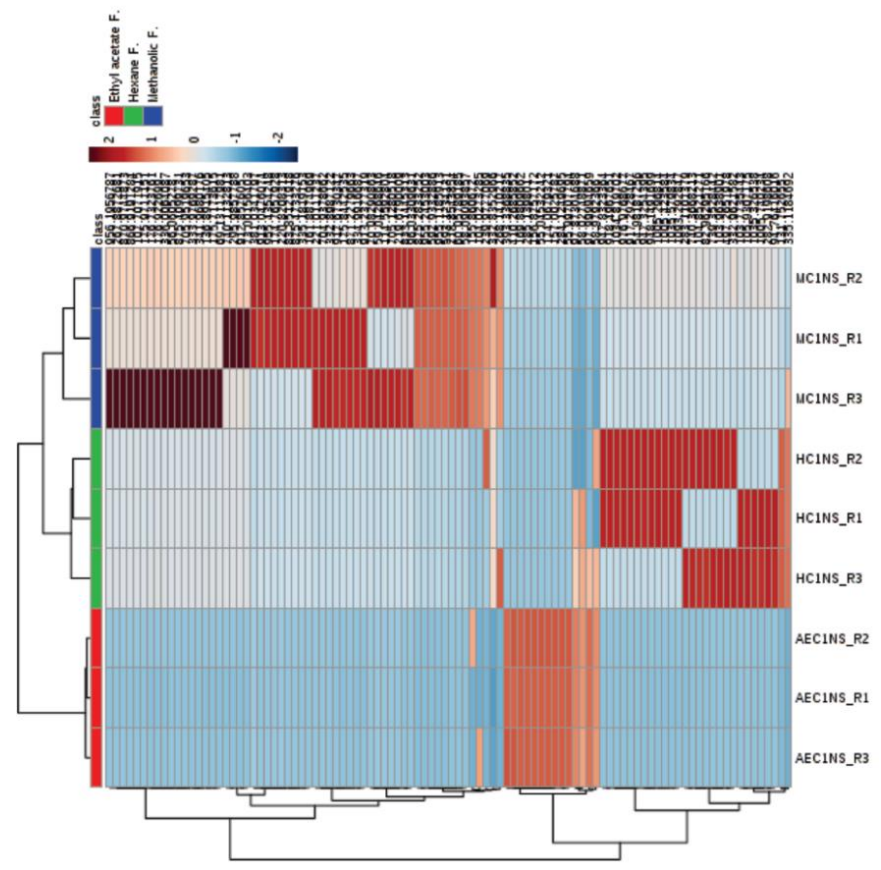

Fig. 3: Heatmap clustering analysis performed in MetaboAnalyst 4. Using DIESI (+) MS spectra of the metabolites obtained in three fractions different of methanolic extracts roots of Baccharis salicina. Analysis was performed using Euclidian distance method with ward clustering algorithm after normalization to sample median and Pareto scaling

\section{Multivariate analysis}

The hierarchical classification by "clusters" can be observed in the graphical representation of a heat map
(Fig. 3). On the right side, there are 100 representative $\mathrm{m} / \mathrm{z}$ of all the samples studied and identified via mass spectrometry. In the lower part of the graph, the fractions in which the crude methanolic extract was partitioned are represented. 
Lam-Gutiérrez et al. / Intl J Agric Biol, Vol 25, No 5, 2021

Table 3: Significant compounds detected in roots of Baccharis salicina by DIESI-MS, negative mode

\begin{tabular}{|c|c|c|c|c|c|c|c|c|}
\hline Fraction & $\begin{array}{l}{[\mathrm{m}-\mathrm{H}]^{-} \quad \mathrm{m} / \mathrm{z}} \\
\text { (ionization mode) }\end{array}$ & Fragment pattern $[\mathrm{m}-\mathrm{H}]\left(\mathrm{MS}^{2}\right)$ & $\begin{array}{l}\text { st intense cor } \\
\text { Ionization } \\
\text { energy }(V)\end{array}$ & $\begin{array}{l}\text { mpounds in } \\
\text { Molecular } \\
\text { formula }\end{array}$ & $\begin{array}{l}\text { Baccharis glutinosa roots } \\
\text { Putative compound }\end{array}$ & Class & Data base & ID data base \\
\hline \multirow[t]{12}{*}{$\begin{array}{l}\text { Ethyl } \\
\text { acetate }\end{array}$} & $174(-)$ & $156,98,116,138,86,69,54$ & 25 & $\mathrm{C}_{7} \mathrm{H}_{10} \mathrm{O}_{5}$ & $\begin{array}{l}\text { (3R,4S,5R)-3,4,5-Trihydroxycyclohex } \\
\text {-1-enecarboxylic acid }\end{array}$ & $\begin{array}{l}\text { Cyclohexanecarboxylic } \\
\text { acid. }\end{array}$ & MoNA & Spectrum PR100485 \\
\hline & $265(-)$ & 96 & 35 & $\mathrm{C}_{18} \mathrm{H}_{18} \mathrm{O}_{2}$ & $\begin{array}{l}\text { 2-(4-hydroxy-3-prop-2-enyl-phenyl)- } \\
\text { 4-prop-2-enyl-phenol }\end{array}$ & $\begin{array}{l}\text { Benzene and substituted } \\
\text { derivatives }\end{array}$ & MoNA & $\begin{array}{l}\text { Spectrum } \\
\text { FiehnHILIC001252 }\end{array}$ \\
\hline & $297(-)$ & $182.92,196.92,211.83,233$ & 30 & $\begin{array}{l}\mathrm{C}_{14} \mathrm{H}_{1} \mathrm{~N} \\
\mathrm{O}_{6}\end{array}$ & $\begin{array}{l}\text { (3,4,5-trihydroxy-6-methyloxan-2-yl) } \\
\text { 2-(methylamino)benzoate }\end{array}$ & $\begin{array}{l}\text { Organooxygen } \\
\text { compounds: } \\
\text { Carbohydrates and } \\
\text { carbohydrate conjugates }\end{array}$ & MoNA & $\begin{array}{l}\text { Spectrum VF-NPL- } \\
\text { QEHF019193 }\end{array}$ \\
\hline & $316(-)$ & $106,298.33,189.25,272.25$ & 30 & $\mathrm{C}_{16} \mathrm{H}_{12} \mathrm{O}_{7}$ & 3'-Methoxyquercetin & Flavonoids: flavonols & MoNA & Spectrum PR100640 \\
\hline & $325(-)$ & $182.92,196.92,169.92,224,261$ & 30 & $\mathrm{C}_{21} \mathrm{H}_{42} \mathrm{O}_{2}$ & Heneicosanoic acid & $\begin{array}{l}\text { Fatty acyls: Fatty acids } \\
\text { and conjugates }\end{array}$ & MoNA & $\begin{array}{l}\text { Spectrum } \\
\text { EXPO_D10_5 } \\
\text { ppm_NEG_iTree }\end{array}$ \\
\hline & $241(-)$ & $171,79.8,145,196.92$ & 25 & $\mathrm{C}_{15} \mathrm{H}_{12} \mathrm{O}_{3}$ & $\begin{array}{l}\text { 2,4-Dihydroxychalcone, también se le } \\
\text { llama 3-(2,4-Dihydroxyphenyl)-1- } \\
\text { phenyl-2-propen-1-one }\end{array}$ & $\begin{array}{l}\text { Phenylpropanoids and } \\
\text { polyketides }\end{array}$ & HMDB & 39612 \\
\hline & $439(-)$ & $\begin{array}{l}337.17,424.25,395,368,323,293.42,162.92 \text {, } \\
176.92\end{array}$ & 25 & $\mathrm{C}_{26} \mathrm{H}_{32} \mathrm{O}_{6}$ & $\begin{array}{l}\text { Oxireno(c)phenanthro(1,2-d)pyran- } \\
\text { 3,8(3aH,4bH)-dione, } \quad 1 \text {-(3-furanyl)- } \\
1,5,6,6 \mathrm{a}, 7,10 \mathrm{a}, 10 \mathrm{~b}\end{array}$ & $\begin{array}{l}\text { Prenol } \\
\text { Triterpenoids }\end{array}$ & MoNA & $\begin{array}{l}\text { Spectrum } \\
\text { NP_C3_114_p2_A05_i } \\
\text { Tree_NEG_46 }\end{array}$ \\
\hline & $410(-)$ & $330.33,258.25,392.33,272.33,244.17,340.25$ & 30 & - & - & - & - & - \\
\hline & $514(-)$ & $353,172.92,203$ & 25 & - & - & - & - & - \\
\hline & $255(-)$ & $175,197,210.92,236.83$ & 25 & - & - & - & - & - \\
\hline & $311(-)$ & $182.92,196.92,247.17,169.92,267.25,280$ & 30 & - & - & - & - & - \\
\hline & $581(-)$ & $501.25,563.17,416.5,374.25$ & 25 & - & - & - & - & - \\
\hline \multirow[t]{13}{*}{ Hexane } & $182.92(-)$ & $138.92,118.92,166.75,155$ & 25 & $\mathrm{C}_{9} \mathrm{H}_{10} \mathrm{O}_{4}$ & 4-O-Methylphloracetophenone & $\begin{array}{l}\text { Organooxygen } \\
\text { compounds: } \\
\text { Phenylketones }\end{array}$ & MoNA & $\begin{array}{l}\text { Spectrum VF-NPL- } \\
\text { QEHF011678 }\end{array}$ \\
\hline & $325.17(-)$ & $182.92,197,169.92 .261 .08,239.08$ & 30 & $\mathrm{C}_{15} \mathrm{H}_{30} \mathrm{O}_{5}$ & $\begin{array}{l}\text { (Z)-3-hydroxy-1-(2-hydroxyphenyl)- } \\
\text { 3-phenylprop-2-en-1-one; tambiéns se } \\
\text { llama 2'-beta-Dihydroxychalcone }\end{array}$ & $\begin{array}{l}\text { Carboxylic acids and } \\
\text { derivatives }\end{array}$ & PubChem & 11688418 \\
\hline & $383(-)$ & $337.42,365.25$ & 30 & $\mathrm{C}_{24} \mathrm{H}_{32} \mathrm{O}_{4}$ & $\begin{array}{l}\text { 5-[(Z)-12-(3,5-dihydroxyphenyl) } \\
\text { dodec-8-enyl]benzene-1,3-diol }\end{array}$ & Phenols: Resorcinols & MoNA & $\begin{array}{l}\text { Spectrum VF-NPL- } \\
\text { QEHF021775 }\end{array}$ \\
\hline & $411(-)$ & $365.42,337.08,382,395.25,349.17,251.08$ & 30 & $\mathrm{C}_{23} \mathrm{H}_{22} \mathrm{O}_{7}$ & 12aß-hydroxydeguelin & Isoflavonoids & MoNA & $\begin{array}{l}\text { Spectrum VF-NPL- } \\
\text { QEHF004037 }\end{array}$ \\
\hline & $142.75(-)$ & $99.83,98.92,110.75,72.75,123,125.17$ & 25 & - & - & - & - & - \\
\hline & $255.25(-)$ & $227,211,196.83,236.92$ & 25 & - & - & - & - & - \\
\hline & $311(-)$ & $482.92,196.92,247.08,169.92,196.92$ & 30 & - & - & - & - & - \\
\hline & $377(-)$ & $219,340.75,303.08,314.92,191,182.92,331.08,159$ & 25 & - & - & - & - & - \\
\hline & $455.33(-)$ & $419.42,393.17,407.33,353.08,332.83$ & 25 & - & - & - & - & - \\
\hline & $499(-)$ & $\begin{array}{l}397.25,382.08,367,484.17,255,453.42,407.67 \text {, } \\
469.08,243.25,271.08,425.25\end{array}$ & 25 & - & - & - & - & - \\
\hline & $671(-)$ & $\begin{array}{l}391.42,256.25,409.17,610.58,627.58,581.5 \text {, } \\
278.233\end{array}$ & 30 & - & - & - & - & - \\
\hline & $281.33(-)$ & $263.08,239.08,209.08,124.92,111,191,252.92$ & 25 & - & - & - & - & - \\
\hline & $325(-)$ & $182.92,310.17,261.17,281,225,238$ & 30 & - & - & - & - & - \\
\hline \multirow[t]{16}{*}{$\begin{array}{l}\text { Methanoli } \\
\mathrm{c}\end{array}$} & $233(-)$ & $143.08,171.08,127,156.83,186.75$ & 25 & $\mathrm{C}_{13} \mathrm{H}_{12} \mathrm{O}_{4}$ & $\begin{array}{l}\text { Goniothalenol o bien 3-hydroxy-2- } \\
\text { phenyl-2,3,3a,7a-tetrahydrofuro[3,2- } \\
\text { b]pyran-5-one }\end{array}$ & Furopyrans & MoNA & $\begin{array}{ll}\text { Spectrum } & \text { VF-NPL- } \\
\text { LTQ004696 }\end{array}$ \\
\hline & $515(-)$ & $353,191,427.58,334.92,317,399,172.75$ & 20 & $\mathrm{C}_{25} \mathrm{H}_{24} \mathrm{O}_{12}$ & 3,5-Dicaffeoylquininic acid & $\begin{array}{l}\text { Organooxygen } \\
\text { compounds }\end{array}$ & MoNA & $\begin{array}{l}\text { Spectrum } \\
\text { CCMSLIB00000081755 }\end{array}$ \\
\hline & $793(-)$ & $\begin{array}{l}647.25,571.42,393.83,585.33,629.25,603.08, \\
553.08\end{array}$ & 25 & $\begin{array}{l}\mathrm{C}_{36} \mathrm{H}_{6} \mathrm{O}_{1} \\
0\end{array}$ & $\begin{array}{l}\text { Olean-12-en-28-oic acid, 3-(beta-D- } \\
\text { glucopyranuronosyloxy)-23-hydroxy- } \\
\text {,(3beta,5xi,9xi,18xi)- }\end{array}$ & Prenol lipids & MoNA & $\begin{array}{l}\text { Spectrum } \\
\text { CCMSLIB0000084998 } \\
6\end{array}$ \\
\hline & $179(-)$ & $134.92,163.92,88.67,68.83$ & 20 & $\mathrm{C}_{9} \mathrm{H}_{8} \mathrm{O}_{4}$ & 3,4-Dihydroxycinnamic acid & $\begin{array}{l}\text { Phenylpropanoids and } \\
\text { polyketides }\end{array}$ & MoNA & Spectrum KO000511 \\
\hline & $311(-)$ & $\begin{array}{l}182.83,250.92,197,280.83,170.92,210.75,140.50 \text {, } \\
122.67\end{array}$ & 35 & $\begin{array}{l}\mathrm{C}_{19} \mathrm{H}_{21} \mathrm{NO} \\
4\end{array}$ & $\begin{array}{l}\text { 1,10-Dimethoxy-6-methyl-5,6,6a,7- } \\
\text { tetrahydro-4H- } \\
\text { dibenzo[de,g]quinoline-2,9-diol }\end{array}$ & Aporphines & PubChem & 248507 \\
\hline & $353(-)$ & $191,178.92,172.92,131,196.92$ & 35 & $\mathrm{C}_{16} \mathrm{H}_{18} \mathrm{O}_{9}$ & $\begin{array}{l}\text { 3-(3,4-Dihydroxycinnamoyl)quinic } \\
\text { acid }\end{array}$ & $\begin{array}{l}\text { Organooxygen } \\
\text { compounds }\end{array}$ & PubChem & 1794427 \\
\hline & $835(-)$ & $585.25,647.33,107.17,817.42,634.75$ & 30 & - & - & - & - & - \\
\hline & $915(-)$ & $835.33,727.17,647.33,629.17,254.92,769.33$ & 30 & - & - & - & - & - \\
\hline & $191(-)$ & $92.83,126.83,108.83,154.83,172.83$ & 20 & - & - & - & - & - \\
\hline & $568(-)$ & $489,416.83,367.25,365.50,527.25,479.17$ & 25 & - & - & - & - & - \\
\hline & $877(-)$ & $585.17,513.25,629.33,471.33,859,479.25$ & 45 & - & - & - & - & - \\
\hline & $369(-)$ & 323.33 & 30 & - & - & - & - & - \\
\hline & $383(-)$ & 337.42 & 30 & - & - & - & - & - \\
\hline & $398(-)$ & $352.33,396.17,382.75$ & 25 & - & - & - & - & - \\
\hline & $472(-)$ & $\begin{array}{l}408.50,444.08,410.25,316.25,454.25,288.33 \text {, } \\
426.58,370,158.92,300,311,324,370\end{array}$ & 25 & - & - & - & - & - \\
\hline & $411(-)$ & 365.33 & 25 & - & - & - & - & - \\
\hline
\end{tabular}

The color scale ( -2 to 2 ) indicates the percentage of relative abundance of the $\mathrm{m} / \mathrm{z}$ detected for each fraction. For equality of observable characteristics, the heat map classified the study fractions into a dendogram. Partitioning the crude extract into three fractions (methanol, hexane and ethyl acetate), allowed to separate metabolites attracted by different polarities. The greatest number of peaks $(\mathrm{m} / \mathrm{z})$ was found to be in the methanolic fraction, followed by hexane and ethyl acetate fractions (Fig. 3).
In total, 539 features in ESI (+) and 119 in ESI (-) mode were found to be statistically significant. The crossvalidation based on the filtered data matrix was performed to estimate the predictive ability of the multivariate Partial Least Squared Discriminant Analysis (PLS-DA) models (Fig. 4). In the score plot, the two main components explain the greater effect of analyzing the methanolic extracts of Chilca roots (B. salicina) inthe three fractions that were considered. The analysis showed the separation of variables 
organized into clusters, in a two-plane graph. The observed trend indicates that, depending on the nature of the fraction, there are characteristic $\mathrm{m} / \mathrm{z}$ for each (Fig. 4A). The main 25 $\mathrm{m} / \mathrm{z}$ identified in positive mode represent the repeated metabolites in the study fractions, considering the Variable Influence on the Projection (VIP) (Fig. 4B).

\section{Tandem mass spectrometry $\left(\mathrm{MS}^{2}\right)$}

The screening analysis allowed a total of 54 compounds to be identified and characterized in both positive and negative mode from MEBs. Fragmentation patterns $\left(\mathrm{MS}^{2}\right)$ were used to determine the identity of each metabolite, based on the most probable molecular mass. This was also verified with on-line libraries and breaking mechanisms. The list of putatively identified compounds is shown in Table 2 and 3.

\section{Discussion}

Loureiro et al. (2015) found $43 \%$ germinated uredospore in the in vitro study on $C$. arabica H147/1 $24 \mathrm{~h}$ after inoculation (Diniz et al. 2012; Yoon et al. 2013) and 34\% in the in vivo condition. In a natural situation, for infection to be completed, spores germinate better on young leaves than on intermediate and old leaves, as they require free water. A temperature between $22-28^{\circ} \mathrm{C}$ is another important factor influencing germination along with moisture. A single lesion on a leaf produces 4 to 6 spore crops over a 3 to 5month period, releasing 300-400,000 spores (Yoon et al. 2013).

Uredospore germination and fungal penetration are key stages for the initial development of the coffee leaf rust pathogen $H$. vastatrix (Talinhas et al. 2016). Fungi infecting from uredospores are particularly sensitive during the initial stages of the disease cycle, especially between spore germination and host penetration (Thines et al. 2004). The presence of antifungal metabolites from MEBs suggests a possible breakdown in the first communications between plant and uredospore.

The growth cycle of $H$. vastatrix is known to begin at the spotting of the host surface, in which uredospores germinate inserting appressoria into the stomata (Talinhas et al. 2016). Therefore, the surface hydrophobicity is an important stimulus for inducing the infection structure in the plant (Kou and Naqvi 2016). It has also been detected at this stage that spores release an adhesive drop from a periplasmic compartment, which attaches the uredospore to hydrophobic surfaces. This glue is likely to contain glycoproteins. It has been observed that plant lectin concavalin A inhibits binding (Thines et al. 2004).

The results presented herein indicate that full inhibition in germination percentage is not achieved. However, there is a trend linking increased treatment doses with reduced germination percentages (Table 1). This means that some important proteins for generating growth could be lost, thus delaying the biotrophic development and growth
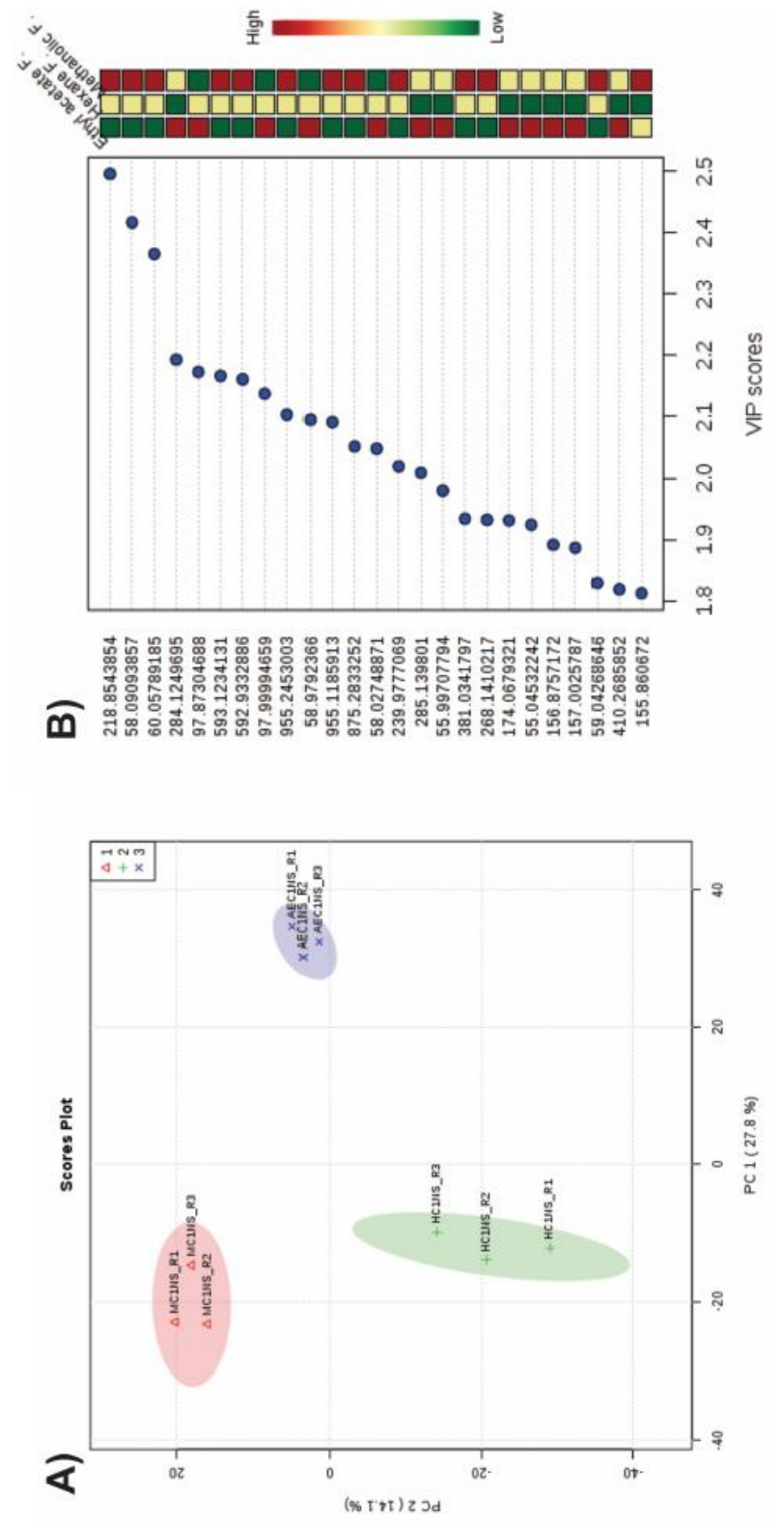

Fig. 4: A) Score plot of the first two principal component of PLSDA using normalization to sample sum and Pareto scaling. B) Significant features identified by PLS-DA performed in MetaboAnalyst 4.0. Positive mode

(Meyer et al. 2009). Deising et al. (2000) and Bölker (1998) reported that chitin deacetylases (CD), G-protein subunits and MAP Kinases (MAPKs) were associated withearly developmental phases of several pathogenic fungi. The genes involved in the early infection process of $H$. vastatrix on $C$. arabica leaves were confirmed by Vieira et al. (2011) with an expression profile (Vieira et al. 2012).

In this study, the flavonoids putatively found were multijuginol, (Z)-3-hydroxy-1-(2-hydroxyphenyl)-3phenylprop-2-en-1-one, 3'-Methoxyquercetin and 12aß- 
hydroxydeguelin. The incidence of 3'-Methoxyquercetin was previously reported in leaves of $B$. pseudotenuifolia by Moreira et al. (2003). In addition, flavonoids, phenolic acids and triterpenes extracted from leaves and roots from $B$. dracunculifolia were reported with immunomodulatory biological activity (Figueiredo-Rinhel et al. 2013; Ramos et al. 2016). An important molecule included in the tetracyclic diterpenoid class are Gibberelines (signaling compounds or plant hormones), which regulate developmental processes such as seed germination, root and shoot elongation, flowering and fruit patterning (Shani et al. 2013). They therefore coordinatethe synthesis of other molecules.In this study, these were detected in roots. In vitro studies have shown that phenolic compounds like flavonoids, coumarines and phenolic acid can have antioxidant activity, which allows them to act as reducing agents (Barchan et al. 2014).

On the other hand, the presence of furans $2,5-$ furandicarboxylic acid, dendrolasin (3-(4,8-dimethyl-3,7nonadienyl)-furan) and 3-hydroxy-2-phenyl-2,3,3a,7atetrahydrofuro[3,2-b]pyran-5-one, coincide with previous work by Lam-Gutiérrez et al. (2019) where this molecule class was found via GC-MS, albeit with adifferent molecular mass. (2E,6E)-2-(4-methylpent-3-en-1-yl)-6-[3(2,5-dihydro-2-oxofuran-3-yl)-propylidene]hept-2-enedioic acid was found in aerial parts from $B$. thymifolia (Hikawczuk et al. 2008). According to Konovalov (2014), a type of polyacetylene commonly spotted in the Asteraceae family is furanopolyacetylene.

In the study of MEBs, compounds such as 3, 4dihydroxycinnamic acid and 3-(3,4-dihydroxycinnamoyl) quinic acid belonging to phenilpropanoids and polyketides class (including hydroxycinnamic acids) could be observed. These kind of molecules have been reported in aerial parts of $B$. grisebachii, B. chilco, B. trimera, B. retusa, B. incarum (leaves and steams) and $B$. dracunculifolia (leaves, stems and roots) (Ramos et al. 2016). García-Jimenez et al. (2018) showed that cinnamic acid, 2-hydroxycinnamic, 2,3 and 4-methoxycinnamic acids were tyrosinase inhibitors. In the same way, it was reported on the fungal $\beta-1,3$-glucanase and chitinase activities of maize phytopathogenic fungi and extracts of aerial parts of B. salicina (Buitimea et al. 2013), was important for the development of fungi. Additionally, Taofiq et al. (2017) found that the derivatives of cinnamic acid had antifungal, antibacterial, antioxidant, antiinflammatory and antitumoral activities.

The type of extraction solvent and its polarity are known to potentially have a significant impact on the number of metabolites extracted (Thouri et al. 2017). Methanol molecules are proton donors, making it the most used solvent in the crude extraction of metabolites, considering a broad-molecule spectrum. Nevertheless, the present study included also a first extraction with methanol, a partition with two other organic solvents that was able to separate metabolites attracted according to the polarity of each one (hexane > ethyl acetate). Hence, the separation of metabolites could be observed. This behavior is largely due to the chemical nature of the organic solvent used. Methanol is a protic polar solvent with the capacity of binding by hydrogen bridges, whereas hexane is non-polar, as it lacks functional groups capable of yielding protons and has a ahigh dielectric constant. On the other hand, ethyl acetate is known for being an aprotic polar solvent and for having a low dielectric constant. Just as hexane, it is lacking in functional groups.

The proposed analysis allowed the finding of major metabolites, which has been suggested as belonging to polyketides (flavonoids, flavonols, isoflavones and chalcones), organoheterocyclic compounds (furans and furopyrans), fatty acyls, prenol-lipids (isoprenoids), organooxygen and farnesene classes. Ramos et al. (2016) reported that the main constituents of Baccharis genus are phenolic and terpenoid compounds, demonstrating that flavonoids and diterpenoids have been the major classes of metabolites, as this study reveals as well. Likewise, antifungal activity in Baccharis genus has been reported in these compounds, as coumarins, triterpenes, sesquiterpenes and flavonoids are present in $B$. darwini (aerial parts), $B$. dracunculifolia (aerial parts), B. elaeoides (leaves), B. semiserrata (twigs) and $B$. retusa (twigs) (Kurdelas et al. 2010; Johann et al. 2012; Vannini et al. 2012; Grecco et al. 2014). Furthermore, antifungals have been reported as being present in roots of B. salicina for the first time. Therefore, the fingerprinting by use of DIESI-MS offers an important tool for pioneering descriptive analysis of metabolites in a reliable screening.

\section{Conclusion}

Concentrations of MEBs $>100 \mathrm{mg} \mathrm{mL}^{-1}$ on leaves of coffee reduce the germination of uredospores from $H$. vastatrix, with significant statistical differences. Using the DIESI-MS tools, a general screening was obtained that allowed the generation of metabolite fingerprints. Greater relative abundance was present in MEBs in an $\mathrm{m} / \mathrm{z}$ range of 50 2000. The previous partition of crude extract methanolic of root from Chilca into three fractions improved the chemical composition study conducted with successive MS-MS analyses, thus identifying an estimated 50 of the most important metabolites. We also speculated briefly about the possible roles of such substances interrupting communication between uredospores and plant in early stages of infection process or reducing synthesis of important proteins which can affect fungal growth and morphological differentiation. Therefore, the study contributes to composition and properties analysis of bioactive phytochemicals from $B$. salicina against coffee leaf rust pathogen $H$. vastatrix. This research should be furthered to better assess the potential activity of B. salicina on multiple other applications in the future.

\section{Acknowledgments}

This research was funded by Project number '6211.17' 
(TecNM, Mexico). The authors wish to thank the 'Instituto Nacional de InvestigacionesForestales, Agrícolas y Pecuarias (INIFAP)'. ALG wishes to acknowledge CONACYT Mexico for her postgraduate scholarship.

\section{Author Contributions}

AL-G and VMR-V conceptualized the work, ERG-R and RW provided laboratory facilities for analyzing the MEBs, RR-R and FAG-M reviewed the manuscript, AL-G statistically analyzed the data, BAP-O and JMG-A analyzed the final version of the graphics.

\section{Conflicts of Interest}

The authors declare that they have no conflicts of interest concerning this article.

\section{Data Availability}

The data will be made avaialble on acceptable requests to the corresponding author.

\section{Ethics Approval}

Not applicable.

\section{References}

Abad MJ, P Bermejo (2007). Baccharis (compositae): a review update. Arkat 7:76-96

Akhtar R, A Javaid, MZ Qureshi (2020). Efficacy of shoot extracts of Sisymbrium irio against Fusarium oxysporum f. spp. cepae. Plant Danin 38; Article e020200961

Barchan A, M Bakkali, A Arakrak, R Pagán, A Laglaoui (2014). The effects of solvents polarity on the phenolic contents and antioxidant activity of three Mentha species extracts. Intl J Curr Microbiol Appl Sci 3:399-412

Bölker M (1998). Sex and crime: heterotrimeric G proteins in fungal mating and pathogenesis. Fung Genet Biol 25:143-156

Buitimea GV, C Rosas, F Cinco, A Burgos, M Plascencia, MO Cortez, JC Gálvez (2013). In vitro effect of antifungal fractions from the plants Baccharis glutinosa and Jacquinia macrocarpa on chitin and $\beta$-1,3-glucan hydrolysis of maize phytopathogenic fungi and on the fungal $\beta-1,3$-glucanase and chitinase activities. J Food Saf 33:526-535

Cox DG, J Oh, A Keasling, KL Colson, MT Hamann (2014). The utility of metabolomics in natural product and biomarker characterization. Biochim Biophys Acta 1840:3460-3474

Deising HB, S Werner, M Wernitz (2000). The role of fungal appressoria in plant infection. Microb Infect 2:1631-1641

Diniz I, P Talhinhas, H Azinheira, V Varzea, C Medeira, I Maia, AS Petitot, M Nicole, D Fernandez, MC Silva (2012). Cellular and molecular analyses of coffee resistance to Hemileia vastatrix and nonhost resistance to Uromyces vignae in the resistance-donor genotype HDT832/2. Eur J Plant Pathol 133:141-157

Figueiredo-Rinhel ASG, LM Kabeya, PCP Bueno, RF Jorge-Tiossi, ECS Azzolini, JK Bastos, YM Lucisano-Valim (2013). Inhibition of the human neutrophil oxidative metabolism by Baccharis dracunculifolia DC (Asteraceae) is influenced by seasonality and the ratio of caffeic acid to other phenolic compounds. J Ethnopharmacol 150:655-664
García-Flores M, S Juárez-Colunga, A García-Cascarrubias, S Trachsel, R Winkler, A Tiessen (2015). Metabolic profiling of plant extracts using direct-injection electrospray ionization mass spectrometry allows for high-throughput phenotypic characterization according to genetic and environmental effects. $J$ Agric Food Chem 63:1042-1052

García-Jimenez A, F García-Molina, JA Teruel-Puche, A Saura-Sanmartin, PA Garcia-Ruiz, A Ortiz-Lopez, JN Rodríguez-López, F GarcíaCasanovas, J Munoz-Munoz (2018). Catalysis and inhibition of tyrosinase in the presence of cinnamic acid and some of its derivatives. Intl J Biol Macromol 119:548-554

Grecco SS, AC Dorigueto, IM Landre, MG Soares, K Martho, R Lima, RC Pascon, MA Vallim, TM Capello, P Romoff, P Sartorelli, JHG Lago (2014). Structural crystalline characterization of sakuranetin - an antimicrobial flavanone from twigs of Baccharis retusa (Asteraceae). Molecules 19:7528-7542

Hikawczuk V, R Saad, O Giordano, T Martín, V Martín, ME Sosa, C Tonn (2008). Insect growth regulatory effects of linear diterpenoids and derivatives from Baccharis thymifolia. J Nat Prod 71:190-194

Javaid A, R Munir, IH Khan, A Shoaib (2020). Control of the chickpea blight, Ascochyta rabiei, with the weed plant, Withania somnifera. Egypt J Biol Pest Cont 30:114-121

Johann S, FB Oliveira, EP Siqueira, PS Cisalpino, CA Rosa, TMA Alves, CL Zani, BB Cota (2012). Activity of compounds isolated from Baccharis dracunculifolia D.C. (Asteraceae) against Paracoccidioides brasiliensis. Med Mycol 50:843-851

Konovalov DA (2014). Polyacetilene compounds of plants of the Asteraceae family (review). Pharm Chem J 48:615-633

Kou Y, N Naqvi (2016). Surface sensing and signaling networks in plant pathogenic fungi. Semin Cell Dev Biol 57:84-92

Kurdelas RR, B Lima, A Tapia, GE Feresin, MG Sierra, MV Rodríguez, S Zacchino, RD Enriz, M Freile (2010). Antifungal activity of extracts and prenylated coumarins isolated from Baccharis darwinii Hook \& Arn. (Asteraceae). Molecules 15:4898-4907

Lam-Gutiérrez A, TR Ayora-Talavera, ER Garrido-Ramírez, FA Gutiérrez-Miceli, JA Montes-Molina, S Lagunas-Rivera, VM Ruíz-Valdiviezo (2019). Phytochemical profile of methanolic extracts from Chilca (Baccharis glutinosa) roots and its activity against Aspergillus ochraceus and Fusarium moniliforme. J Environ Biol 40:302-308

López-Bravo DF, EDM Virginio-Filho, J Avelino (2012). Shade is conductive to coffee rust as compared to full sun exposure under standardized fruit load conditions. Crop Prot 38:21-29

Loureiro A, H Azinheira, M Silva, PA Talhinhas (2015). Method obtaining from Hemileia vastatrix appresoria produced in planta, suitable for transcriptomic analyses. Fungal Biol 119:1093-1099

McCain JW, JF Hennen (1984). Development of the uredinial thallus and sorus in the orange coffee rust fungus, Hemileia vastatrix. Phytopathology 74:714-721

Meyer V, M Arentshorst, S Flitter, BM Nitsche, MJ Kwon, C ReynagaPeña, S Bartnicki-García, CVD Hondel, A Ram (2009). Reconstruction of signaling networks regulating fungal morphogenesis by transcriptomics. Eukariotic Cell 8:1677-1691

Moreira FPM, V Voutinho, AB Pimentel, MSP Caro, IM Costa, M Pizzolatti, F Delle (2003). Flavonoids and triterpenes from Baccharis pseudotenuifolia - Bioactivity on Artemia salina. Quim Nova 26:309-311

Naqvi SF, A Javaid, MZ Qureshi (2019). Evaluation of antifungal potential of leaf extract of Chenopodium murale against Fusarium oxysporum f. spp. lycopersici. Plant Danin 37; Article e019199050

Ramos F, J Bressan, VC Godoy, T Zuccolotto, LED Silva, L Bonancio (2016). Baccharis (Asteraceae): chemical constituents and biological activities. Chem Biodivers 13:1-17

Rosas-Burgos EC, MO Cortez-Rocha, M Plascencia-Jatomea, FJ CincoMoroyoqui, RE Robles-Zepeda, J López-Cervantes, DI SánchezMachado, F Lares-Villa (2011). The effect of Baccharisglutinosa extract on the growth of mycotoxigenic fungi and fumonisin B1 and aflatoxin B1 production. World $J$ Microbiol Biotechnol 27:1025-1033 
Shani E, R Weinstain, Y Zhang, C Castillejo, E Kairseli, J Chory, RY Tsien, M Estelle (2013). Gibberellins accumulate in the elongating endodermal cells of Arabidopsis root. Proc Natl Acad Sci USA 12:4834-4839

Talinhas P, D Batista, I Diniz, A Vieira, D Silva, A Loureiro, S Tavares, AP Pereira, HG Azinheira, L Guerra-Guimaraes, V Várzea, MC Silva (2016). The Coffee leaf rust pathogen Hemileia vastatrix: one and a half centuries around the tropics. Mol Plant Pathol 18:1039-1051

Taofiq O, A González-Paramás, MF Barreiro, IC Ferreira (2017). Hydroxycinnamic acids and their derivatives: cosmeceutical significance, challenges and future perspectives, a review. Molecules 22:281-304

Tequida-Meneses M, M Cortez-Rocha, EC Rosas-Burgos, S LópezSandoval, C Corrales-Maldonado (2002). Efecto de extractos alcohólicos de plantas silvestres sobre la inhibición de crecimiento de Aspergillus flavus, Aspergillus niger, Penicillium chrysogenum, Penicillium expansum, Fusarium moniliforme y Fusarium poae. Rev Iberoam Micol 19:84-88

Thines E, H Anke, R Weber (2004). Fungal secondary metabolites as inhibitors of infection-related morphogenesis in phytopathogenic fungi. Mycol Res 108:14-25
Thouri A, H Chahdoura, A El-Arem, A Omri-Hichri, R Ben-Hassin, L Achour (2017). Effect of solvents extraction on phytochemical components and biological activities of Tunisian date seeds (var. Korkobbi and Arechti). BMC Complem Altern Med 17; Article 248

Vannini AB, TG Santos, AC Fleming, LRP Purnhagen, LA Lourenzo, ETB Butzke, M Kempt, IM Begnini, RA Rebelo, EM Dalmarco, AB Cruz, AP Schmit, RCB Cruz, CN Yamanaka, M Steindel (2012). Chemical characterization and antimicrobial evaluation of the essential oils from Baccharis uncinella DC and Baccharis semiserrata DC (Asteraceae). J Essent Oil Res 24:547-554

Vieira A, P Talhinhas, A Loureiro, J Thürich, S Duplessis, D Fernandez, MC Silva, OS Paulo, H Azinheira, (2012). Expression profiling of genes involved in the biotrophic colonization of Coffea arabica leaves by Hemileia vastatrix. Eur J Plant Pathol 133:261-277

Vieira A, P Talinhas, A Loureiro, S Duplessis, D Fernandez, MC Silva, OS Paulo, H Azinheira (2011). Validation of RT-qPCR reference genes for in planta expression studies in Hemileia vastatrix, the causal agent of coffee leaf rust. Fung Biol 115:891-901

Xia J, DS Wishart (2016). Using MetaboAnalyst 3.0 for comprehensive metabolomics data analysis. Curr Protoc Bioinform 55:14-10

Yoon MY, B Cha, JC Kim (2013). Recent trends in studies on botanical fungicides in agriculture. Plant Pathol 29:1-9 